Adhesive forces 6

Adipose tissue 101

Anchored disc phenomenon 6

Anti-AQP-1 antibody 117

Antigen presentation 184

Antiresorbing agents 17

Apoptosis 34

Aquaporin 117

Arthritic pain 49

Arthritis 26

Basic calcium phosphate crystals 63

Biotinylated dextran amine immunotracer 194

Blood-epididymis barrier 162

Blood-testis barrier 162

Bone resorption 17

Cattle 170

Cell culture system 155

CHC1-L 155

Chick 194

Chinchilla, nasal cavity 136

Chondrocytes, human 34

Collagens 170

Cytokine 34

Dendritic cells 184

Differentiation, monocytes/macrophages 110

Dog 162

Early growth response gene 63

Fiber types 73

Friction 6

GABA immunostaining 194

Gene expression 63

Golgi architecture 194

Granuloma 110

\section{Heart 184}

Hyaluronan 49

Hydrostatic pressure 155

Hylan 49

Hypercholesterolemia 184

Inflammation 26

Intercellular space 162

Internal derangement 6

Intranasal immunization 136
Lipoaspirate 101

Liver 117

Lymphocyte 155

Macrophage(s) 110, 184

Masseter 87

Mesenchymal stem cell 101

Mononuclear cells, primary human 110

Motoneurons 87

Multicellular spheroid 110

Muscle 73

Myosin 73

Nasal-associated lymphoid tissue 136

Neuroblastoma 155

Nitric oxide 26

Nucleus lentiformis mesencephali magnocellularis 194

Open lock 6

Osteoarthritis 6,34

Osteoclast 17

Osteoporosis 17

Otitis media 136

Pig 117

Placenta 170

Processed lipoaspirate cells 101

Rabbit 184

Reverse-transcription polymerase chain reaction 73

RGS5 155

Sexual dimorphism 87

Signaling pathways 34

Skull growth 129

Smooth muscle cells 162

Temporomandibular joint $6,17,49$

- _ disease 17,26

Testosterone 87

Tissue culture, three-dimensional 110

Transcriptional factors 63

Transgenerational undernutrition 129

Tumor necrosis factor 26

Uterus 170

Viscosupplementation 49

\section{KARGER}

(c) 2003 S. Karger AG, Base

Fax + 41613061234

E-Mail karger@karger.ch

www.karger.com
Accessible online at: www.karger.com/cto 